\title{
A Comparison of the Capacities of VR and 360- Degree Video for Coordinating Memory in the Experience of Cultural Heritage
}

\author{
Shengdan Cai \\ Faculty of Humanities and Social \\ Sciences \& NVIDIA Joint-Lab on \\ Mixed Reality \\ University of Nottingham \\ Ningbo, China \\ shengdan.cai@nottingham.edu.cn
}

\author{
Eugene Ch'ng \\ Faculty of Humanities and Social \\ Sciences \& NVIDIA Joint-Lab on \\ Mixed Reality \\ University of Nottingham \\ Ningbo, China \\ eugene.chng@nottingham.edu.cn
}

\author{
Yue Li \\ International Doctoral Innovation \\ Centre \& NVIDIA Joint-Lab on Mixed \\ Reality \\ University of Nottingham \\ Ningbo, China \\ yueli@nottingham.edu.cn
}

\begin{abstract}
Virtual Reality (VR), a medium which can create alternate or representations of reality, could potentially be used for triggering memory recollections by connecting users with their past. Comparing to commonly-used media within museum such as photos and videos, VR is distinct because of its ability to move beyond the confines of time and space, by enabling users to be immersed in the reconstructed context and allowing them to take charge of the environment by interacting with objects, navigating the environment, and evolving the narratives. In this paper, we compared audience experiences of cultural heritage $(\mathrm{CH})$ between 360-degree video recordings and Virtual Environments to investigate the capacity of these two types of media for coordinating the audience's memory of the past. The findings will help guide the future design and evaluation of $V R$ as a medium for communicating $\mathrm{CH}$.
\end{abstract}

Keywords - Cultural Heritage, Virtual Reality, 360-degree Video, Memory, The Past

\section{INTRODUCTION}

Cultural heritage $(\mathrm{CH})$ encapsulates historical information and memories, which are intrinsic to individuals who are familiar with it, and also recorded as texts and probably within pictures or other media, and therefore viewing and interacting with them will recall memories for those individuals. When it comes to VR, will the virtually presented and represented $\mathrm{CH}$ impact on individual's memory recollections and reconstruction remains to be answered.

\section{BACKGROUND}

\section{A. Representation of Cultural Heritage}

There is a long history of using different media to record and represent $\mathrm{CH}$ - text, oral, paint, photo - often exercise "transformative power" over what is being communicated [1]. Dave [1] has stated the transformation of this "power", from the text and paint media, which was dominated by human subjectivity, to print and photography media, which achieved "exactly repeatable statement", to virtual technologies which "emphasise evidence, veracity of detail and relativist interpretations in place of a singular history". However, virtual technology was initially an instrument of statically and accurately reproducing and reconstructing three-dimensional models of heritage sites and landscapes for historic research, which to some extent was no different from the repeatable photographic representation. As it moved from the realm of scholarly research into public institutions, a decade of works have seen the exploration of virtual representation of $\mathrm{CH}$ from the perspectives of public users: engagement, interactivity, sense of place, and etc. [2]-[4].

There is an area lacking needed research, that is, how well different modes of communicating $\mathrm{CH}$ are, i.e., videos, images, text, if compared to VR. We believe that this article pioneers this area by comparing VR to 360-degree videos, which are already more vivid than 2D video and images [5].

\section{B. Relationship between Memory and Virtual Heritage}

While the association between memory and heritage has long been recognised, their relationship in the virtual environment has not been explored. Memory by nature is selective, fragmented, and unstable, which thus needs to be embodied and structuralised within material objects and other concrete forms of culture [6], [7]. Nora [8] argued that the real environments of memory ("milieux de mémoire") will irresistibly fade away with the passage of time, as a consequence, "lieux de mémoire" with material, symbolic and functional features such as objects and places, become the only way through which modern society collectively remembers. VR technologies are able to reconstruct or recontextualise the "lieux de mémoire" in the virtual environments. Very few studies considered the role of $\mathrm{CH}$ delivered by virtual means within people's memory. Silberman [9] assigned more importance to understand why the past is important than what the past is, and highlighted the necessity of integrating the broader role of $\mathrm{CH}$ in society into the development of virtual heritage, otherwise it is just "a storehouse of visually presented object" [10].

Our study virtually represents the dwellings where the everyday life of local Ningbonese people has practiced since the last century, a near past where memories are still living, and related artefacts are still available for digital capture. Our initial hypotheses are that 1) $\mathrm{CH}$ delivered by VR can trigger the recollection and reconstruction of memory for those familiar with the objects and scenes; 2) VR has greater capacity for coordinating memory in the experience of local $\mathrm{CH}$ as compared to the 360-degree video.

\section{Methodology}

\section{A. 360-Degree Video \& Virtual Environment}

The high-resolution 360-degree video (3840 x 1920) for experiment captured the interior of a well-preserved house located in a village of Ningbo and recorded an old couple cooking food. This video was captured by Samsung Gear 360 camera positioned at the centre of the house on a tripod. 
The participants wore the Samsung Gear VR headset (SMR322) to watch the video (see Fig. 1.).

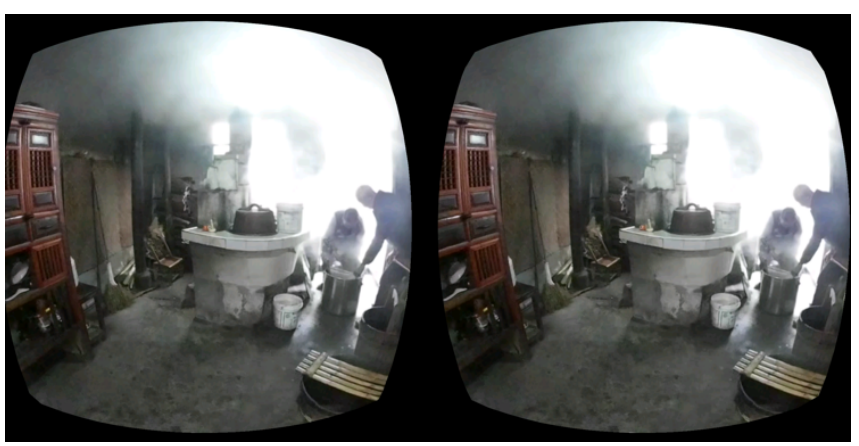

Fig. 1. User view for left and right eyes (video length: $8 \mathrm{~m} 26 \mathrm{~s}$ )

For the virtual "house", we assembled the models of artefacts captured from the reality to make the past reappeared in the virtual environment. These models were developed from photogrammetry models generated by Autodesk ReCap Photo and RealityCapture with the photos collected from fieldtrips to villages and heritage sites. The raw models were retopologised and texture-baked within Blender and exported to Unity for virtual environment development. The models were enhanced further with texture and normal mapping to achieve a visual quality asymptotic to the original object. The detailed workflow is described in Fig. 2. When objects were not practical for photogrammetry, or difficult to find or captured in real life, we modelled them within Blender based on the photos collected, and rendered them with real-world textures for realism.

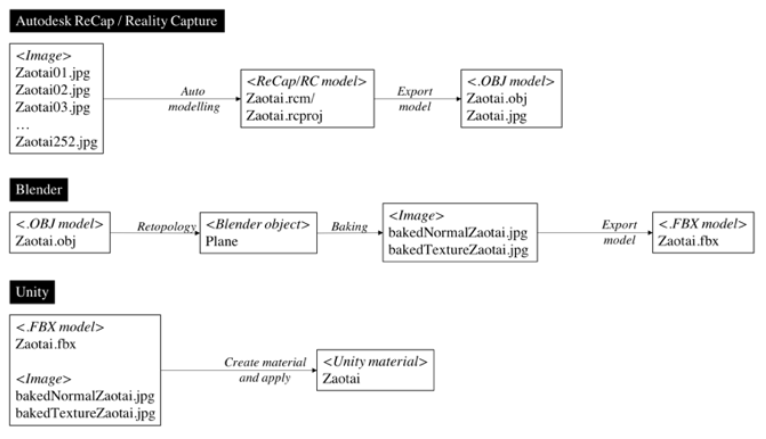

Fig. 2. Process workflow of cultural heritage object

The virtual "house" (see Fig. 3.) was developed in Unity (version 2018.1.6f1) built on a professional VR workstation with NVIDIA Quadro M6000 24GB graphics card and HTC VIVE kit (one headset, two sensors and two hand-held controllers). Interactions in virtual environments were designed with reference to real-life activities, which include opening drawers, grabbing objects, and etc.
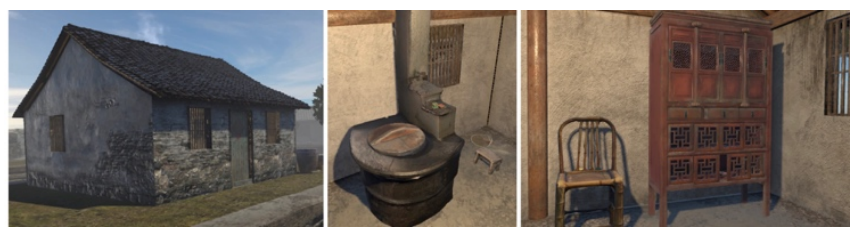

Fig. 3. The virtual "house" (left), Zaotai (the cooking stove, middle), chair and cupboard (right)

\section{B. Participant Study}

\section{1) Demographics Group for Participant Study}

We randomly recruited 21 participants (7 males, 14 females) through the university's emails, posters, and social media posts. All the participants are local residents (i.e. born and raised in Ningbo, and their parents and grandparents are all Ningbonese), as they were assumed to be familiar with the local history and customs. There are 12 out of 21 participants (57.14\%) aged between 18-24, and 7 of them $(33.33 \%)$ aged between $25-34$. We have 1 participant from the age group of 45-54 and 1 participant aged above 75 .

\section{2) Equipment and Test Environment}

The experiment took place at the NVIDIA Joint-Lab on Mixed Reality at the University of Nottingham's China campus. A $3 \times 3 \mathrm{~m}$ VR experience area was set up, allowing participants to physically walk around the space. The sequence of the two experiences was randomised to mitigate the possibility of a medium influencing the other.

\section{Data Collections}

\section{1) Non-participant Observation}

An observation sheet was used for recording the time each participant spent in each experience, and their reactions and actions (e.g. facial, verbal, behavioural expressions) that can be observed within the physical environment.

\section{2) Questionnaire Survey}

All participants filled in a questionnaire with three sections, these include: a) questions about demographic information and prior experience; b) 7-point Likert scale questions related to user experience (1 being "strongly agree" 7 being "strongly disagree"). The questions were adapted from established Nostalgia, and VR questionnaires ([2], [11]-[13]); c) 7-point Likert scale questions that allow participants to compare their experience with 360-degree video to the virtual environment.

Each participant was required to fill in the Section a before the experience, answer the questions in the Section $b$ after experiencing the virtual environment and again after watching the 360 -degree video. Section $c$ is completed at last.

\section{3) Semi-structured Interviews}

Open-ended questions were asked in the interviews, which included 1) the influences of each experience on memories (e.g., what do you think triggered familiarity); 2) comparison between the video and VR (e.g., which ones do you prefer and why); 3) expectations (e.g., what else did you expect to have in the video and the virtual environment); 4) alternative questions related to each one's personal real-life experience and behaviour during the experience.

\section{Data Analysis}

For the quantitative data collected from instrument recordings, and closed-ended questions, Plotly's Python graphing library was used for analysing and visualising the datasets. The qualitative data generated from the observation notes and interviews were thematically interpreted and analysed with NVivo in order to capture underlying ideas and nuanced perception of participants. 


\section{A. Memory Was Recalled}

An overview of our participants' feedback on their experience with both systems is presented in Table I. According to the Mean and the Standard Deviation of their responses, most participants "felt a sense of familiarity" with both scenes $(\mu=2.05, \sigma=0.67$ for video and $\mu=2.29, \sigma=0.72$ for VR). The participants also tended to agree that "the scenes reminded of an experience I had from the past" ( $\mu=2.05, \sigma=0.74$ for video and $\mu=2.52, \sigma=0.87$ for VR), indicating that both scenes have successfully recalled their memories of the past. This finding is further confirmed by the participants' negative responses to the $\mathrm{q} 5$ and $\mathrm{q} 7$. Moreover, the positive answers to $\mathrm{q} 3(\mu=2.57, \sigma=1.16$ for video and $\mu=2.81, \sigma=0.75$ for $V R$ ) indicated that the participants not only triggered memory recollections but had a sense of nostalgia. The experiences of local $\mathrm{CH}$ through both media have provided the participants with a sense of familiarity, recalled the memories of the past, and induced a collection of reminiscence.

\section{TABLE I. DESCRIPTIVE DATA I}

\begin{tabular}{|c|c|c|c|c|c|c|}
\hline & & $N$ & (video) & (video) & $(V R)$ & $(V R)$ \\
\hline q1 & I felt a sense of familiarity with the scene. & 21 & 2.05 & 0.67 & 2.29 & 0.72 \\
\hline q2 & The scene reminded me of an experience I had from the past. & 21 & 2.05 & 0.74 & 2.52 & 0.87 \\
\hline $\mathrm{q}^{3}$ & I missed this past experience that the scene reminded me of. & 21 & 2.57 & 1.16 & 2.81 & 0.75 \\
\hline $\mathrm{q} 4$ & $\begin{array}{l}\text { The scene made me recall an experience, and I now feel sad } \\
\text { because the happy memory is over. }\end{array}$ & 21 & 3.48 & 1.21 & 3.81 & 1.21 \\
\hline q5 & The scene did not give me any feelings about the past. & 21 & 5.33 & 1.11 & 5.33 & 1.02 \\
\hline q6 & $\begin{array}{l}\text { I wish I could relive the experience(s) the scene made me think } \\
\text { of. }\end{array}$ & 21 & 3.62 & 1.36 & 3.38 & 1.12 \\
\hline q7 & I did not think about the past when I visited the scene. & 21 & 5.43 & 1.16 & 5.33 & 1.06 \\
\hline q8 & I was transported to the past. & 21 & 2.81 & 1.17 & 3.00 & 1.22 \\
\hline q9 & $\begin{array}{l}\text { When I was in the scene, my heart did beat faster as it was like } \\
\text { visiting somewhere I had been before. }\end{array}$ & 21 & 3.19 & 1.29 & 3.76 & 1.41 \\
\hline q10 & When I was in the scene, I could smell things from my past. & 21 & 2.71 & 1.01 & 2.81 & 1.08 \\
\hline q11 & When I was in the scene, I could hear things from my past. & 21 & 2.43 & 0.75 & 3.67 & 1.39 \\
\hline q12 & I felt pleasant when I was in the scene. & 21 & 2.81 & 1.25 & 2.29 & 0.85 \\
\hline q13 & I felt warm when I was in the scene. & 21 & 2.33 & 1.02 & 2.38 & 0.80 \\
\hline q14 & I felt a sense of peacefulness when I was in the scene. & 21 & 2.52 & 1.21 & 2.71 & 1.27 \\
\hline q15 & I felt a sense of sadness when I was in the scene. & 21 & 4.81 & 1.33 & 4.95 & 1.16 \\
\hline q16 & I felt a sense of regret when I was in the scene. & 21 & 4.71 & 1.52 & 4.29 & 1.49 \\
\hline q17 & I felt anxious when I was in the scene. & 21 & 5.14 & 1.46 & 5.14 & 1.28 \\
\hline q18 & I had a sense of belonging when I was & 21 & 2.71 & 1.10 & 2.76 & 0.83 \\
\hline
\end{tabular}

Comparing the participants' feedbacks on the two sets of questions (see Fig. 4.), we observed that in terms of the sense of familiarity and the recollection of memory, more participants rated the 360-degree video as slightly higher than VR. This view is also confirmed by the disagreement that most participants expressed with q19 and q20 (see Table II \& Fig. 5.). The participants explained their rating in the interviews, that the 360-degree video made them feel more real as they believed it was "a video record of reality" (p19).

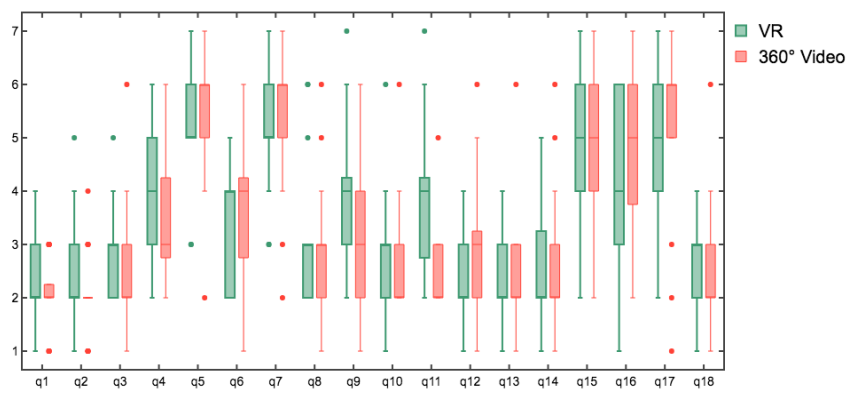

Fig. 4. Participants' feedbacks on two experiences
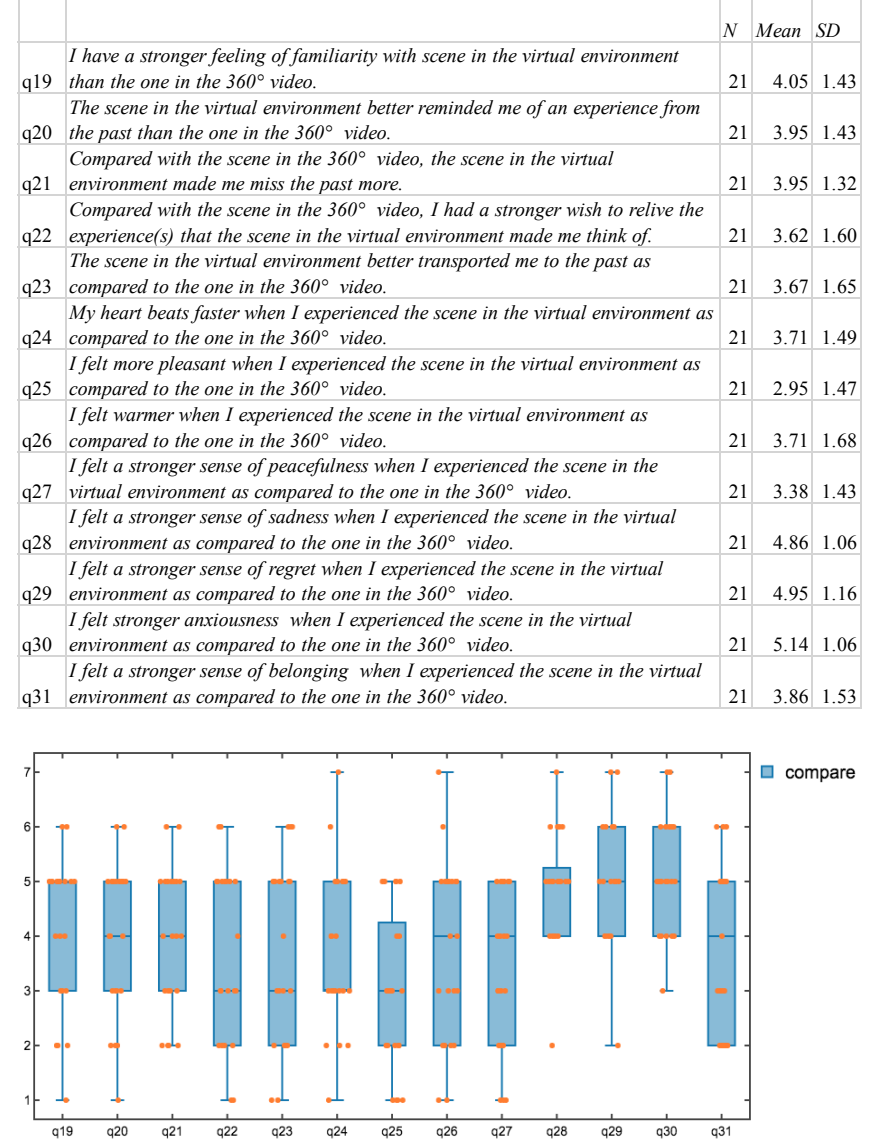

Fig. 5. Participants' evaluation of statements comparing the video and VR

We cannot arbitrarily conclude that the 360-degree video has a greater capacity for triggering people's memories of the past. It is due to the fact that when asked what triggered familiarity and their memories in the interviews, most participants referenced the objects within the virtual environment to answer the question, and rarely mentioned the 360-degree video. In most cases, the participants compared the objects from the virtual environment with the old objects they remembered from the past, such as "the wooden bench and the cupboard were the same as the ones in my grandma's house" (p13); "my grandma also used this brand of matchsticks" (p11).

Also, the participants were more likely to mention the objects by associating a particular meaning to them, and spontaneously related these items with their memories of peoples, activities and sentiments from the past. For example, "cupboard was used to put tableware, my grandma used it every day" (p18): "all my families came to my grandma's house in the Chinese New Year, she was busy preparing the food around Zaotai" (p13); "taste of the rice cooked in Zaotai was really good” (p7).

Given all these responses, it is undeniable that the virtual environment has highly contributed to the familiarity and memory recollections, even though the 360-degree video were thought to be more real.

\section{B. Exploring and Participating in the Past Instead of Watching the Past}

We triangulated the data collected from the questionnaire, observations and interviews, and discovered that the length of time most participants spent in the virtual 
environment was far more than the time they spent in the video (see Fig. 6.). Only 3 out of 21 participants spent 5-10 minutes in the 360 -degree video, 8 of them spent $2-5$ minutes, 7 participants spent 1-2 minutes and 3 of them spent less than one minute. It is less likely that the participants have fully explored the video within such a short time.

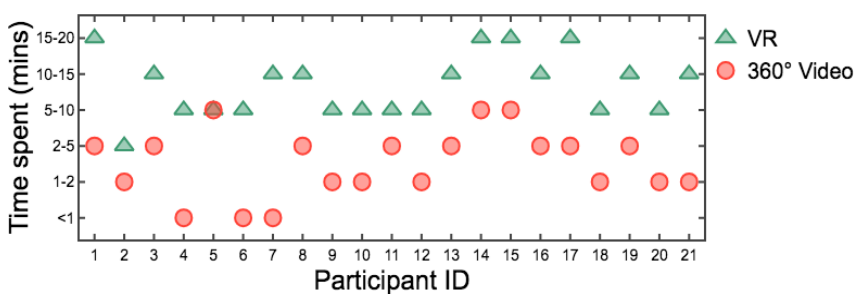

Fig. 6. Time spent in the virtual environment and the 360-degree video

The novelty of the VR technology could be one of the factors that prolonged the duration of the VR experience. Two-thirds of our participants $(n=14)$ were attracted by VR and thereby enrolled in our study, although more than $80 \%$ $(n=17)$ of our participants have never experienced 360degree videos, as compared to $57 \%(\mathrm{n}=12)$ for VR.

Another factor is that the participants were more willing to learn, explore and interact within the virtual environment than watch the 360-degree video. The participants have highlighted that it was the actual people in the video and the sound that provided them with a sense of reality. We quoted some of the responses below: "The sound of the firewood burning, and the old couples were talking to each other. I felt I went back to my grandparents' house, watching them cook together, just in the same way I did when I was a child" (p2); "I could hear that they were talking in the local dialect" (p8); "I could feel that a real life is going on there" (p1\&p14). Meanwhile, the participants also expressed their disappointment of being a passive spectator in the 360degree video, such as "I wish I could get a step closer to see what they were cooking" (p15); "It was like my soul was transported to the past, but my body was not. But for VR, both my soul and body have travelled back to the past" (p8).

As a result, some participants have articulated their preferences for the VR experience because they have greater freedom to "define the ways in which I would like to interact with the objects and with the environment" (p14). Also, the participants have proactively proposed common ideas to further develop the virtual environment with the reference of their memories about the past, such as hawkers on the street, family activities in festive seasons. They believed that VR technology has greater potentials than 360-degree videos if any such technologies were to be used for remembering the past. Exploring and participating in the past with VR will increase audience attention, thus with a longer-lasting impact on the recollections and reconstructions of memory, as compared to watching the past in the 360 -degree video.

\section{CONCLUSION}

This study compared the potentials of different media types for triggering memory recollections in the experience of local $\mathrm{CH}$. Here, we conclude that both 360-degree video recordings and true VR reconstructions of local $\mathrm{CH}$ can trigger a sense of familiarity, nostalgia, and recollections of past memories. We demonstrated that 360-degree video has the advantage of providing a sense of reality, which stems from its full video footage of scenes, sounds and activities from reality. 360-degree videos make a spectator passive, in contrast, virtual environments allow a higher degree of freedom in which individuals are able to explore and interact with the past. This impact upon, prolongs and reinforces the memory recollection and reconstruction. Our work is a very first such study on comparing 360-degree videos to true VR environments, and the findings thus contribute to the future design and evaluation of $\mathrm{VR}$ as a medium for communicating $\mathrm{CH}$. It extends the theoretical framework of $\mathrm{CH}$ and studies on memory to the virtual realm. Virtual time travel [14] may just have become a real possibility.

\section{ACKNOWLEDGMENT}

This study was supported by the Faculty of Humanities and Social Sciences, and International Academy of Marine Economy and Technology of University of Nottingham Ningbo China, and Zhejiang Science and Technology Development Commonwealth Grant. The study was carried out at the NVIDIA Joint-Lab on Mixed Reality.

\section{REFERENCE}

[1] B. Dave, "Virtual Heritage: Mediating space, time and perspectives," in New Heritage: New media and cultural heritage, Y. E. Kalay, T. Kvan, and J. Affleck, Eds. Oxon: Routledge, 2008, pp. 40-52.

[2] P. Turner and S. Turner, "Place , Sense of Place and Presence," Presence Teleoperators Virtual Environ., vol. 15, no. 2, pp. 204-217, 2006.

[3] L. Pujol and M. Economou, "Exploring the suitability of Virtual Reality interactivity for exhibitions through an integrated evaluation: the case of the Ename Museum," Online Int. Museol. J., vol. 4, pp. 84-97, 2007.

[4] E. Champion and B. Dave, "Dialing Up the Past," in Theorizing Digital Cultural Heritage A Critical Discourse, F. Cameron and S. Kenderdine, Eds. Cambridge: The MIT Press, 2007, pp. 333-348.

[5] J. Bailenson, K. Patel, A. Nielsen, R. Bajscy, S. H. Jung, and G. Kurillo, "The effect of interactivity on learning physical actions in virtual reality," Media Psychol., vol. 11, no. 3, pp. 354-376, 2008.

[6] A. Radley, "Artefacts, memory and a sense of the past," in Collective Remembering, D. Middleton and D. Edwards, Eds. London: Sage Publications, 1990, pp. 46-59.

[7] J. Urry and J. Larsen, The Tourist Gaze. SAGE Publications Ltd, 2012.

[8] P. Nora, "Between Memory and History: Les Liex de Mémoire," Representations, vol. 26, pp. 7-25, 1989.

[9] N. Silberman, "Chasing the unicorn?: The quest for 'essence' in digital heritage," in New Heritage: New media and cultural heritage, Y. E. Kalay, T. Kvan, and J. Affleck, Eds. Oxon: Routledge, 2008, pp. 81-91.

[10] J. Preece, D. Benyon, G. Davies, L. Keller, and R. Rogers, A Guide to Usability: Human Factors in Computing. Wokingham, England: Reading, MA, Addison-Wesley, 1993.

[11] A. Merchant, K. LaTou, J. B. Ford, and M. S. LaTour, "How strong is the pull of the past? measuring personal nostalgia evoked by advertising," J. Advert. Res., vol. 53, no. 2, 2013.

[12] S. M. Baker and P. F. Kennedy, "Death by Nostalgia: a Diagnosis of Context-Specific Cases," Adv. Consum. Res., vol. 21, pp. 169-174, 1994.

[13] M. Usoh, E. Catena, S. Arman, and M. Slater, "Using Presence Questionnaires in Reality," Presence Teleoperators Virtual Environ., vol. 9, no. 5, pp. 497-503, 2000.

[14] E. Ch'ng, "Experiential Archaeology: Is Virtual Time Travel Possible?," J. Cult. Herit., vol. 20, pp. 458-470, 2009. 